\title{
Leches a base de plantas: Cáñamo'
}

\author{
Sarah Curl, Daniela Rivero-Mendoza, y Wendy J. Dahl²
}

La leche de cáñamo es una leche de origen vegetal que está ganando popularidad. La semilla de cáñamo comercial, utilizada en la producción de leche de cáñamo, contiene solo trazas del compuesto tetrahidrocannabinol (THC), el cual es demasiado bajo para producir efectos psicoactivos por el consumo de la leche (Rodríguez-Leyva et al. 2010). Esta publicación describe la composición de la leche de cáñamo y los posibles beneficios y riesgos para la salud.

\section{¿Cómo se elabora la leche de cáñamo?}

La leche de cáñamo comercial se elabora combinando la semilla de cáñamo con agua (Wang et al. 2018). Primero, las semillas de cáñamo se mezclan con agua caliente; estas se hinchan y ablandan a medida que absorben agua (Mitchell et al. 2008). Esta mezcla caliente de semillas de cáñamo en agua se muele hasta que se alcanza la consistencia deseada (Mitchell et al. 2008). La lechada filtrada se homogeneiza (se hace uniforme) para mayor estabilidad y luego se enfría para detener la oxidación (pérdida) de los aceites y vitaminas (Mitchell et al. 2008). Se agregan agua, emulsionantes, estabilizadores, edulcorantes y saborizantes para personalizar la leche de cáñamo (Mitchell et al. 2008). Finalmente, se esteriliza con el tratamiento a temperatura ultra alta (UHT), el cual se lleva a cabo para extender la vida útil del producto en la alacena (Mitchell et al. 2008).

\section{¿Cómo se compara el perfil de nutrientes de la leche de cáñamo con el de la leche de vaca?}

La composición de nutrientes de la leche de cáñamo y la leche de vaca se muestra en la Tabla 1. En esta comparación, la leche de cáñamo no tiene azúcar agregado, no está saborizada y está fortificada con calcio. Una porción de leche de cáñamo sin azúcar agregado y que no está saborizada proporciona alrededor de 60 calorías, menos que una porción de leche de vaca. La leche de cáñamo proporciona aproximadamente 3 gramos (g) de proteína por porción, mientras que la leche de vaca proporciona $8 \mathrm{~g}$. La leche de cáñamo es más alta en grasas monoinsaturadas y poliinsaturadas que la leche de vaca baja en grasa y sin grasa (USDA n.d.). Una porción de leche de cáñamo sin azúcar no contiene carbohidratos, mientras que las variedades endulzadas pueden contener más de $20 \mathrm{~g}$ por porción, la mayoría de los cuales son azúcar agregado (Pacific Foods n.d.-a). En comparación, la leche de vaca proporciona 12 $\mathrm{g}$ de carbohidratos proveniente del azúcar lactosa natural. Una taza de leche de cáñamo sin azúcar proporciona menos potasio, pero cantidades similares de sodio y calcio (cuando están fortificadas) a las de la leche de vaca. Algunas leches de cáñamo también están fortificadas con vitaminas $\mathrm{A}, \mathrm{D}$, B2 y B12 (USDA n.d.). Es importante revisar la etiqueta de información nutricional o la lista de ingredientes para determinar si la leche de cáñamo está fortificada.

1. This document is FS431, one of a series of the Food Science and Human Nutrition, UF/IFAS Extension. Original publication date May 2021. Visit the EDIS website at https://edis.ifas.ufl.edu for the currently supported version of this publication.

2. Sarah Curl, former graduate student, Food Science and Human Nutrition Department; Daniela Rivero-Mendoza, Extension and research coordinator; and Wendy J. Dahl, associate professor and Extension specialist, Food Science and Human Nutrition Department, UF/IFAS Extension; Gainesville, FL 32611.

The Institute of Food and Agricultural Sciences (IFAS) is an Equal Opportunity Institution authorized to provide research, educational information and other services only to individuals and institutions that function with non-discrimination with respect to race, creed, color, religion, age, disability, sex, sexual orientation, marital status, national origin, political opinions or affiliations. For more information on obtaining other UF/IFAS Extension publications, contact your county's UF/IFAS Extension office. U.S. Department of Agriculture, UF/IFAS Extension Service, University of Florida, IFAS, Florida A \& M University Cooperative Extension Program, and Boards of County Commissioners Cooperating. Nick T. Place, dean for UF/IFAS Extension. 


\section{¿Cuáles son los posibles beneficios para la salud de la leche de cáñamo?}

Las semillas de cáñamo son uno de los pocos alimentos de origen vegetal que tiene niveles adecuados de todos los aminoácidos esenciales para ser considerada una proteína completa (House et al. 2010). Además, las semillas de cáñamo son ricas en grasas omega-3 (USDA n.d.); los niveles más altos de grasas omega- 3 están asociados con la salud humana (Casula et al. 2020). Muy poca investigación ha examinado específicamente otros beneficios para la salud de consumir leche de cáñamo. En un pequeño estudio, se demostró que el aceite de cáñamo mejora la dermatitis atópica (Callaway et al. 2005). Se cree que esto se debe al equilibrio de grasas inflamatorias y antiinflamatorias. La inflamación es un proceso normal de curación de tejidos; sin embargo, la inflamación excesiva o prolongada puede dañar la piel y provocar dermatitis (Innes et al. 2018). Los niveles de grasas esenciales, que se ha descubierto que brindan beneficios para la salud y la apariencia de la piel (Instituto Linus Pauling de la Universidad Estatal de Oregón sin fecha), aumentaron en las membranas de las células de la piel después de la suplementación con aceite de cáñamo (Callaway et al. 2005). Tanto la sequedad como la picazón de la piel mejoraron después de la intervención con aceite de cáñamo (Callaway et al. 2005). Sin embargo, este estudio se realizó con aceite de cáñamo; se necesita investigación para probar los efectos del consumo de leche de cáñamo sobre la inflamación y la salud de la piel.

Las semillas de cáñamo se están estudiando en relación con las enfermedades cardiovasculares. La proteína de la semilla de cáñamo tiene niveles más altos de arginina (un aminoácido) (Rodríguez-Leyva et al. 2010), que, una vez consumida, se transforma en óxido nítrico, una sustancia que relaja los vasos sanguíneos y mejora la circulación (Wells et al. 2005). La arginina se ha asociado con niveles más bajos de proteína $\mathrm{C}$ reactiva (un marcador de inflamación), que se asocia con enfermedades cardíacas e inflamación crónica (Wells et al. 2005). Sin embargo, es importante tener en cuenta que la leche de cáñamo contiene bajas cantidades de semillas de cáñamo por porción, por lo que el nivel de arginina en la leche de cáñamo es bajo y, por lo tanto, podría no tener ningún efecto protector contra las enfermedades cardiovasculares.

Uno de los beneficios de la leche de cáñamo es que es apropiada para las alergias o intolerancias a la leche de vaca, ya que no contiene proteínas de la leche ni lactosa, el azúcar de la leche. Por lo tanto, las personas alérgicas a las proteínas de la leche de vaca, o con algún grado de intolerancia a la lactosa, pueden consumir leche de cáñamo con seguridad. El cáñamo no es uno de los ocho alérgenos alimentarios principales (FDA 2021), una ventaja sobre la leche de almendras y de soja. Sin embargo, la leche de cáñamo no es nutricionalmente equivalente a la leche de vaca.

La leche de cáñamo es una fuente de grasas insaturadas. Las pautas alimentarias para los estadounidenses recomiendan reemplazar las grasas saturadas por grasas insaturadas porque pueden reducir el riesgo de enfermedad cardiovascular (USDA 2015).

\section{¿Cuáles son los posibles riesgos de la leche de cáñamo?}

Una desventaja de la leche de cáñamo es su bajo nivel de proteínas por porción. Por lo tanto, las personas deben ser conscientes de que, si eligen consumir leche de cáñamo en lugar de leche de vaca, deben obtener la proteína adecuada de otras fuentes o mejorar el contenido de proteína de la leche de cáñamo agregando una proteína en polvo, como proteína de guisante (alverjas) o soja.

\section{¿Es la leche de cáñamo una opción adecuada para los niños?}

El consumo adecuado de proteínas es especialmente importante para los niños y adolescentes. El crecimiento infantil se asocia negativamente con el reemplazo de la leche de vaca con alternativas a la leche de origen vegetal, posiblemente debido a una menor ingesta de proteínas (Morency et al. 2017). Se alienta a los niños mayores de 2 años que no consumen leche de vaca a que beban leches vegetales fortificadas con al menos $6 \mathrm{~g}$ de proteína por ración (Sethi et al. 2016), lo que excluye la leche de cáñamo. Además, algunas leches de cáñamo en el mercado no están fortificadas con vitaminas A o D y, por lo tanto, no son comparables a otras leches de origen vegetal o de vaca como fuentes de estas vitaminas. Además, la forma de calcio utilizada para fortificar la leche de cáñamo puede tener una biodisponibilidad menor que la proporcionada en la leche de vaca (Bridges 2018, Singhal et al. 2017).

\section{Resumen}

Puede haber beneficios para la salud del aceite de semilla de cáñamo, pero no se sabe si la leche de cáñamo proporciona algún beneficio para la salud. Al igual que con otras leches de origen vegetal, la leche de cáñamo, aunque es más baja en calorías y una fuente de grasas saludables, es mucho más baja en proteínas que la leche de vaca. La leche de 
cáñamo no debe ser un sustituto completo de la bebida de la leche de vaca en los niños y puede que no sea una opción adecuada para los adultos mayores que tienen mayores necesidades de proteínas.

\section{Referencias}

Bridges, Meagan. 2018. "Moo-Ove over, Cow's Milk: The Rise of Plant-Based Dairy Alternatives." Practical Gastroenterology 21. Accessed September 30, 2020. https://practicalgastro.com/wp-content/uploads/2019/07/Moo-ove-OverCow-Milk-Rise-of-Plant-Based-Dairy-Alternatives.pdf

Callaway, James, Ursula Schwab, Ilkka Harvima, Pirjo Halonen, Otto Mykkänen, Pekka Hyvönen, and Tomi Järvinen. 2005. "Efficacy of Dietary Hempseed Oil in Patients with Atopic Dermatitis." Journal of Dermatological Treatment 16 (2): 87-94. https://doi.org/10.1080/09546630510035832

Casula, Manuela, Elena Olmastroni, Marta Gazzotti, Federica Galimberti, Alberto Zambon, and Alberico L. Catapano. 2020. "Omega-3 Polyunsaturated Fatty Acids Supplementation and Cardiovascular Outcomes: Do Formulation, Dosage, and Baseline Cardiovascular Risk Matter? An Updated Meta-analysis of Randomized Controlled Trials." Pharmacological Research 160:105060. https://doi.org/10.1016/j.phrs.2020.105060

Food and Drug Administration (FDA). 2021. "Food Allergies: What You Need to Know." Accessed May 3, 2021. https://www.fda.gov/food/buy-store-serve-safe-food/ food-allergies-what-you-need-know

House, James, Jason Neufeld, and Gero Leson. 2010. "Evaluating the Quality of Protein from Hemp Seed (Cannabis sativa L.) Products through the Use of the Protein Digestibility-Corrected Amino Acid Score Method." Journal of Agricultural and Food Chemistry 58 (22): 11801-7. https://doi.org/10.1021/jf102636b

Innes, Jacqueline K., and Philip C. Calder. 2018. "Omega-6 Fatty Acids and Inflammation." Prostaglandins Leukot Essent Fatty Acids 132:41-48. https://doi.org/10.1016/j. plefa.2018.03.004

Mitchell, Pat R., and Khalid M. Shammet. 2008. Hemp Food Product Base and Processes. Google Patents.

Morency, Marie-Elssa, Catherine S. Birken, Gerald Lebovic, Yang Chen, Mary L'Abbé, Grace J. Lee, Jonathon L. Maguire, and the TARGet Kids! Collaboration. 2017. "Association between Noncow Milk Beverage Consumption and Childhood Height." The American Journal of Clinical Nutrition 106 (2): 597-602. https://doi.org/10.3945/ ajcn.117.156877

Oregon State University Linus Pauling Institute. n.d. "Essential Fatty Acids and Skin Health." Accessed September 30, 2020. https://lpi.oregonstate.edu/mic/health-disease/ skin-health/essential-fatty-acids\#overview

Pacific Foods. n.d.-a. "Hemp Vanilla." Accessed September 30, 2020. https://www.pacificfoods.com/our-products/ hemp-plant-based-beverages/hemp-vanilla/

Pacific Foods. n.d.-b. "Unsweetened Hemp Original." Accessed September 30, 2020. https://www.pacificfoods. com/our-products/hemp-plant-based-beverages/ unsweetened-hemp-original/

Rodriguez-Leyva, Delfin, and Grant N. Pierce. 2010. "The Cardiac and Haemostatic Effects of Dietary Hempseed." Nutrition \& Metabolism 7 (1): 32. https://doi. org/10.1186/1743-7075-7-32

Sethi, Swati, S. K. Tyagi, and Rahul K. Anurag. 2016. "Plant-Based Milk Alternatives an Emerging Segment of Functional Beverages: A Review." Journal of Food Science and Technology 53 (9): 3408-3423. https://doi.org/10.1007/ s13197-016-2328-3

Singhal, Sarita, Robert D. Baker, and Susan S. Baker. 2017. "A Comparison of the Nutritional Value of Cow's Milk and Nondairy Beverages." Journal of Pediatric Gastroenterology and Nutrition 64 (5): 799-805. https://doi.org/10.1097/ mpg.0000000000001380

United States Department of Agriculture (USDA). 2015. "Dietary Guidelines for Americans 2015-2020." Accessed September 30, 2020. http://health.gov/ dietaryguidelines/2015/guidelines/

United States Department of Agriculture (USDA). n.d. Fooddata Central. Accessed September 30, 2020. https://fdc. nal.usda.gov/index.html

Wang, Qingling, Jiang Jiang, and Youling L. Xiong. 2018. "High Pressure Homogenization Combined with $\mathrm{Ph}$ Shift Treatment: A Process to Produce Physically and Oxidatively Stable Hemp Milk." Food Research International 106:487-494. https://doi.org/10.1016/j.foodres.2018.01.021 
Wells, Brian J., Arch G. Mainous, and Charles J. Everett.

2005. "Association between Dietary Arginine and C-

Reactive Protein." Nutrition 21 (2): 125-130. https://doi.

org/10.1016/j.nut.2004.03.021

Tabla 1. Perfil de nutrientes de la leche de cáñamo en comparación con la leche de vaca sin grasa, baja en grasa y entera.

\begin{tabular}{|c|c|c|c|c|}
\hline & $\begin{array}{l}\text { Leche de cáñamo sin } \\
\text { azúcar ( } 1 \text { taza) }\end{array}$ & $\begin{array}{c}\text { Leche sin grasa } \\
\text { (descremada) } \\
\text { (1 taza) }\end{array}$ & $\begin{array}{l}\text { Leche baja en grasa (1\%) } \\
\qquad(1 \text { taza) }\end{array}$ & $\begin{array}{l}\text { Leche entera } \\
\text { (1 taza) }\end{array}$ \\
\hline Energía (calorías) & 60 & 83 & 102 & 150 \\
\hline Proteína (g) & 3 & 8 & 8 & 8 \\
\hline Grasa total (g) & 4.5 & 0 & 2.4 & 8 \\
\hline Grasa saturada (g) & NR & 0.1 & 1.5 & 4.5 \\
\hline Carbohidratos (g) & 0 & 12 & 12 & 12 \\
\hline Fibra (g) & 0 & 0 & 0 & 0 \\
\hline Azúcares totales (g) & 0 & 12 & 13 & 12 \\
\hline Vitamina A (mcg) & NR & 149 & 142 & 112 \\
\hline Vitamina B12 (mcg) & NR & 1 & 1 & 1 \\
\hline Vitamina D (mcg) & 2 & 3 & 3 & 3 \\
\hline Calcio (mg) & 257 & 298 & 305 & 276 \\
\hline Sodio (mg) & 110 & 102 & 107 & 105 \\
\hline Potasio (mg) & 100 & 381 & 366 & 322 \\
\hline
\end{tabular}

NR: No reportado; Fuente: USDA (n.d.), Pacific Foods (n.d.-b) 